\title{
Interpretation of pressuremeter data in cohesive soil by simplex algorithm
}

\author{
A.-B. HUANG, J.-L. CHAMEAU and R. D. HOLTZ (1986). Géotechnique 36, No. 4, 599-603
}

\author{
Dr J. M. Kirby, CSIRO Division of Soils, Can- \\ berra
}

The simplex algorithm is well known as a direct and efficient tool for optimization and function minimization (of which curve fitting is a particular example). The Authors propose the use of the simplex algorithm as a standard technique for pressuremeter test data interpretation. However, in making this proposal the Paper fails to discuss several points that should be considered in a standard method.

Firstly, there is more than one simplex algorithm (see Nguyen (1985) for discussion on this point and also for an interesting application in slope stability analysis). The algorithm used by the Authors is the same as a computer program listed as one of their references (Caceci \& Cacheris, 1984). However, there is a need to compare the method described with alternatives. More importantly, the Authors should have discussed the advantages of simplex algorithms over other methods, and the problems which can be experienced in its use. Both these points are discussed by Caceci \& Cacheris (1984).

The main advantages of the simplex algorithm over other methods are that it is simple to program and is fast in operation; no great knowledge of mathematics is required (unlikc many other minimization routines neither derivatives nor matrix operations are required); finally, divergence is impossible.

There are several problems in the use of a simplex algorithm. Firstly, where a function has several minima, the simplex will stop in the first that it reaches. To guard against this it is advisable to run the program several times with different starting positions. For example the Denby equation listed by the Authors possesses minima in 'imaginary' space where one or more of the parameters has an impossible value $\left(G_{i}<0\right.$ etc.). The simplex cannot enter this space (the Denby equation cannot be evaluated for $G_{i}<0$ when $\left.\varepsilon_{r 0}>\tau_{u} / 2 G_{i}(1-2 f)\right)$ and it is usual to incorporate rules constraining the simplex to remain in allowable space. However, should the simplex be started on a slope towards such an imaginary minimum, it will move towards it and finish against the constraint. Thus while divergence is impossible, convergence is not guaranteed. The choice of size for the initial simplex should also be considered. Too large a simplex can cause an unstable leap towards an impossible region of space; too small a size may increase the number of iterations required, depending on the type of simplex algorithm used.

When the region near a minimum (local or global), or sometimes a saddle point, has been found, premature conclusion is still possible depending on the convergence criteria. This happens on a shallow slope when the values of the function at the simplex vertices are sufficiently close together to fulfil the convergence criteria. In proposing a standard method, the choice of convergence criteria should be fully discussed.

Problems can arise when the sums of squares surface is ill conditioned in the sense that the contours of equal sums of squares are very elongate. The best fit parameters may be difficult to obtain on such an ill-conditioned surface because many other possible values for the parameters are nearly as good. Ill-conditioned surfaces may reflect either a model with too many parameters or a poor choice of scaling of the parameters. These problems are common to all iterative nonlinear estimation techniques. Further information on 'overparameterization' and 'reparameterization' can be found in Draper \& Smith (1981) (who refer to other iterative techniques for nonlinear estimation, but not to the simplex method).

In performing a regression, it is useful to quote the significance level of the fit in addition to the standard deviation of the errors as done by the Authors. While the Arnold equation clearly does not fit the data in the Paper as well as the other equations, it is not possible to judge from the Paper whether the fit is significantly worse. Significance levels can be evaluated either by a Monte-Carlo technique (see Caceci \& Cacheris (1984) or the 'bootstrap' method of Diaconis \& Efron (1983)) or by an analysis of variance. The Authors also imply that if 'rogue' data points are in excess of one point in 20 then the results of the regression will change; they miss the point that it is the change in significance level which is important in such a case.

The simplex algorithm is a versatile tool for 
curve fitting, function minimization and optimization. It is applicable to a wide variety of problems in soil mechanics. However, like many other numerical methods, its use requires careful consideration of the pitfalls and the results. This is particularly so when it is proposed as a standard method.

\section{REFERENCES}

Caceci, M. S. \& Cacheris, W. P. (1984). Fitting curves to data: the simplex algorithm is the answer. Byte 9 , No. $5,340-362$.

Diaconis, P. \& Efron, B. (1983). Computer intensive methods in statistics. Scient. Am. 248, 116-130.

Draper, N. R. \& Smith, H. (1981). Applied regression analysis, 2nd edn, Ch. 10. Wiley: New York.

Nguyen, V. U. U. (1985). Determination of critical slope failure surfaces. J. Geotech. Engng Div. Am. Soc. Civ. Engrs 111, GT2, 238-250.

\section{Authors' reply}

The Authors are in complete agreement with the Writer regarding the advantages of the simplex method as well as the necessity for careful consideration of the applicability of numerical methods whenever they are used. Ilowever, it was neither the purpose nor within the scope of the Paper to discuss advantages and disadvantages of the simplex algorithm. This is left to specialized publications or textbooks such as those referred to by Dr Kirby.

To prevent the algorithm from searching into an impossible space (e.g. negative $G$ or $s_{\mathrm{u}}$ etc.), flags were placed in the HSIMPLE program. An extremely large value of the sum of the squares of residuals SSR was assigned when the vertex jumped into such an impossible space. This forced the vertex to reflect out of it.

A consideration of significance levels is certainly an improvement. One obvious way to evaluate them is to compare the derived soil parameters for different simplex-generated optimum vertices. For the example presented in the Paper, the curve fit by the Arnold equation is significantly poorer, because the $s_{\mathrm{u}}$ value is approximately $25 \%$ higher than obtained by the others (with lower SSRs). In the interpretation of pressuremeter data, the Authors always perform a few simplex optimization runs using different sets of initial vertices which are randomly selected. This will generate several optimum vertices and their corresponding SSRs. The best among them is then selected.

The Authors agree that, like most numerical techniques, the simplex algorithm suffers from limitations, and thus the proposed evaluation technique is not perfect. However, the Authors believe that at present it is significantly better than the currently available methods.

Finally, the indices of the weights in equation (1) in the Paper are not correct. The corrected equation is

$$
\begin{aligned}
\mathrm{SSR}= & \sum_{i=1}^{n_{1}} W_{i}\left(\mathrm{f}_{1}\left(X_{i}\right)-y_{i}\right)^{2}+\sum_{n_{1}+1}^{n_{2}} W_{i}\left(\mathrm{f}_{2}\left(X_{i}\right)-y_{i}\right)^{2} \\
& +\ldots+\sum_{i=n_{k-1}+1}^{N} W_{i}\left(\mathrm{f}_{\mathrm{k}}\left(X_{i}\right)-y_{\mathrm{i}}\right)^{2}
\end{aligned}
$$

